\title{
On the $k$-invariants of iterated loop spaces
}

\author{
Dominique Arlettaz \\ Institut de mathématiques, Université de Lausanne, $\mathrm{CH}-1015$ Lausanne, \\ Switzerland
}

(MS received 26 February 1988. Revised MS received 29 April 1988)

\section{Synopsis}

The purpose of this paper is to give universal bounds for the order of the Postnikov $k$-invariants of infinite loop spaces. This is done by giving universal bounds for the order of the $k$-invariants of $m$-connected $r$-fold loop spaces in dimensions $\leqq r+2 m$. An application of the result provides information on the Hurewicz homomorphism between the algebraic $K$-theory of a ring and the homology of its general linear group.

\section{Introduction}

Let $X$ be a connected simple $C W$-complex and let $X[n]$ denote the $n$-th Postnikov section of $X: X[n]$ is a $C W$-complex obtained from $X$ by adjoining cells of dimension $\geqq n+2$, such that $\pi_{i} X[n]=0$ for $i>n$ and $\pi_{i} X[n] \cong \pi_{i} X$ for $i \leqq n$. The Postnikov $k$-invariants $k^{n+1}(X)$ of $X$ are maps $X[n-1] \rightarrow K\left(\pi_{n} X, n+1\right)$ and thus cohomology classes in $H^{n+1}\left(X[n-1] ; \pi_{n} X\right)$, for $n \geqq 2$. All spaces we consider in this paper are connected $C W$-complexes.

It is well known that the $k$-invariants of $H$-spaces of finite type (i.e. having finitely generated integral homology groups in each dimension) are of finite order (cf. [7, Théorème 6] or [1, Proposition 4.1]). We are interested in the order of the $k$-invariants of iterated loop spaces, but we do not assume that the spaces we are looking at are of finite type. In [2, Section 1] we have proved that the order of the $k$-invariants of connected infinite loop spaces is finite and we have given upper bounds for them. The purpose of the present paper is to provide a more general and more precise result.

We consider $r$-fold iterated loop spaces instead of infinite loop spaces and, in Section 1 , we define positive integers $S_{j}$ (for $j \in \mathbb{Z}$ ) with the following property:

THEOREM 0 . If $X$ is an $m$-connected $r$-fold loop space $(m \geqq 0, r \geqq 0)$, then

$$
S_{n-m} k^{n+1}(X)=0
$$

for $2 \leqq n \leqq r+2 m$.

This assertion produces a universal bound, $S_{n-m}$, for the order of the $k$-invariant $k^{n+1}(X)$ of all $m$-connected $r$-fold loop spaces $X$, assuming $2 \leqq n \leqq$ $r+2 m$.

In Section 2 we show that, if we restrict our attention to simply connected spaces, the bound $S_{n-m}$ is the "best possible" in the following sense: let $m \geqq 1$ and $n \geqq 2$ be given integers; then, for each prime $p$ dividing $S_{n-m}$, there exists an $m$-connected infinite loop space $X$ such that its $k$-invariant $k^{n+1}(X)$ is an element 
of order $p$ in $H^{n+1}\left(X[n-1] ; \pi_{n} X\right)$ (cf. Theorem 2.1). We consider also the case where $m=0$ : Theorems 2.4 and 2.6 determine the "best" upper bound (in the same sense) for the order of the $k$-invariant $k^{n+1}(X)$ when $X$ ranges over all connected $r$-fold loop spaces (for $2 \leqq n \leqq r$ ), but it turns out that this bound is in general smaller than $S_{n}$.

Our results provide in particular (the "best") universal bounds for the order of all $k$-invariants of infinite loop spaces (cf. Corollaries 1.9 and 2.5); they are better than those given in [2, Theorem 3].

Finally, we study some consequences of our main theorems in Section 3. For instance, we obtain information on the Hurewicz homomorphism between the algebraic $K$-theory of a ring $R$ and the homology of its general linear group (cf. Corollary 3.4).

\section{Universal bounds for the order of the $\boldsymbol{k}$-invariants of iterated loop spaces}

In this section we want to establish our main result (Theorem 1.6): we shall exhibit universal bounds for the order of the first $k$-invariants of iterated loop spaces. We start by recalling the following assertion on the stable homology of Eilenberg-MacLane spaces.

Definition 1.1 . For $j \geqq 1$, let $M_{j}$ denote the product of all prime numbers $p \leqq(j / 2)+1\left(M_{1}=1\right)$.

LEMMA 1.2. For any abelian group $G$ and for each integer $s \geqq 2$ one has

$$
M_{i-s} H_{i}(K(G, s) ; \mathbb{Z})=0
$$

for $s<i<2 s$.

Proof. This is a direct consequence of [4, Exposé 11, Théorème 2].

In order to generalise this result, we look at spaces with finitely many non-trivial homotopy groups.

DefinItION 1.3. $N_{j, h}:=\prod_{k=0}^{h} M_{j-k}$, for $j>h \geqq 0$.

LemMA 1.4. Let $X$ be an $(s-1)$-connected $C W$-complex $(s \geqq 2)$ and assume there exists an integer $t \geqq s$ such that $\pi_{i} X=0$ for $i>t$. Then

$$
N_{i-s, t-s} H_{i}(X ; \mathbb{Z})=0 \text {, }
$$

if $t<i<2 s$.

Proof. Let $i$ be an integer satisfying $t<i<2 s$. If $t=s$, then $X$ is an Eilenberg-MacLane space and the result is given by Lemma 1.2. Thus we may suppose $t>s$. For $k=1,2, \ldots, t-s$, we consider the fibration

$$
K\left(\pi_{s+k} X, s+k\right) \rightarrow X[s+k] \rightarrow X[s+k-1],
$$

whose Serre spectral sequence provides the exact sequence

$$
H_{i}\left(K\left(\pi_{s+k} X, s+k\right) ; \mathbb{Z}\right) \rightarrow H_{i}(X[s+k] ; \mathbb{Z}) \rightarrow H_{i}(X[s+k-1] ; \mathbb{Z}),
$$

since $i<2 s$. Observe that for $k=1, X[s+k-1]=X[s]=K\left(\pi_{s} X, s\right)$, and recall that, by Lemma $1.2, M_{i-s} H_{i}(X[s] ; \mathbb{Z})=0$ and $M_{i-s-k} H_{i}\left(K\left(\pi_{s+k} X, s+k\right) ; \mathbb{Z}\right)=0$ 
for $k=1,2, \ldots, t-s$. We conclude by induction that $\boldsymbol{M}_{i-s} \boldsymbol{M}_{i-s-1} \ldots$ $M_{i-t} H_{i}(X[t] ; \mathbb{Z})=0$. Because $X[t]=X$, we have $N_{i-s, t-s} H_{i}(X ; \mathbb{Z})=0$.

Since the $k$-invariant $k^{n+1}(X)$ of a $C W$-complex $X$ belongs to the cohomology of a space with finitely many non-trivial homotopy groups, $X[n-1]$, the previous lemma enables us to prove our main result.

Definition 1.5. $S_{j}:=\prod_{k=2}^{j} M_{k}$ for $j \geqq 2$ and $S_{j}:=1$ for $j \leqq 1$.

When $j \geqq 2$, note that a prime number $p$ divides $S_{j}$ if and only if $p \leqq \frac{j}{2}+1$; therefore the product of all prime factors of $S_{j}$ is $M_{j}$.

Theorem 1.6. Let $X$ be an $m$-connected $C W$-complex ( $m \geqq 0)$. If $X$ is an $r$-fold loop space $(r \geqq 0)$, i.e. $X \simeq \Omega^{r} Y$ for some $C W$-complex $Y$, then

for $2 \leqq n \leqq r+2 m$.

$$
S_{n-m} k^{n+1}(X)=0 \text {, }
$$

(The statement of this theorem is valid without any finiteness condition on $X$.)

Proof. Because $X$ is $m$-connected, it is clear that $k^{n+1}(X)=0$ for $2 \leqq n \leqq$ $m+1$. Thus, we may consider dimensions $n$ with $m+2 \leqq n \leqq r+2 m$ (in particular, we suppose $r+m \geqq 2$ ). It follows from the homotopy equivalence $X \simeq \Omega^{r} Y$ that $\pi_{n} X \cong \pi_{n+r} Y$ and that the $k$-invariants of $X$ are related to those of $Y$ by the formula

$$
\left(\sigma^{*}\right)^{r}\left(k^{n+r+1}(Y)\right)=k^{n+1}(X),
$$

where $\left(\sigma^{*}\right)^{r}: H^{n+r+1}\left(Y[n+r-1] ; \pi_{n} X\right) \rightarrow H^{n+1}\left(X[n-1] ; \pi_{n} X\right)$ is the $r$-fold iterated cohomology suspension $[9$, p. 438]. Note that we may assume $Y$ is $(m+r)$-connected. Applying Lemma 1.4 to the space $Y[n+r-1]$ by setting $s=m+r+1$ and $t=n+r-1 \quad(t \geqq s \geqq 3$ because $n \geqq m+2, r+m \geqq 2)$ we obtain:

$$
N_{i-m-r-1, n-m-2} H_{i}(Y[n+r-1] ; \mathbb{Z})=0,
$$

for $n+r-1<i<2 m+2 r+2$, in particular for $i=n+r$ and $i=n+r+1$ since $n \leqq r+2 m$. Therefore, we deduce from the universal coefficient theorem that the group $H^{n+r+1}\left(Y[n+r-1] ; \pi_{n} X\right)$ has finite exponent which is bounded by $\operatorname{lcm}\left(N_{n-m-1, n-m-2}, N_{n-m, n-m-2}\right)=\operatorname{lcm}\left(M_{1} M_{2} \ldots M_{n-m-1}, M_{2} M_{3} \ldots M_{n-m}\right)=S_{n-m}$. Consequently, $S_{n-m} k^{n+r+1}(Y)=0$ and

$$
S_{n-m} k^{n+1}(X)=\left(\sigma^{*}\right)^{r}\left(S_{n-m} k^{n+r+1}(Y)\right)=0 .
$$

Remark 1.7. In the case $r=0$, the assertion of the theorem is the following: if $X$ is an $m$-connected $C W$-complex (with $m \geqq 1$ ), then

$$
S_{n-m} k^{n+1}(X)=0, \quad \text { for } 2 \leqq n \leqq 2 m .
$$

Remark 1.8. This result (for $r=0$ ) is wrong if $n>2 m$ : in this case, $k^{n+1}(X)$ may be of infinite order. Assume for instance that $n=2 m+1$ where $m$ is an odd integer, and take a generator $\alpha$ of the infinite cyclic direct summand of the group $H^{2 m+2}(K(\mathbb{Z}, m+1) ; \mathbb{Z})$; then consider $\alpha$ as a map $K(\mathbb{Z}, m+1) \rightarrow K(\mathbb{Z}, 2 m+2)$, and call $X$ its fibre: $X$ is an $m$-connected space with only two non-trivial 
homotopy groups and its unique $k$-invariant is $k^{n+1}(X)=\alpha$, which is of infinite order. For this reason the argument of the proof of Theorem 1.6 is false without the hypothesis $n \leqq r+2 m$ : the order of the $k$-invariant $k^{n+r+1}(Y)$ may be infinite if $n>r+2 m$.

The conclusion of Theorem 1.6 produces in particular universal bounds for the order of all $k$-invariants of infinite loop spaces:

CoROllary 1.9. If $X$ is an $m$-connected infinite loop space, then

$$
S_{n-m} k^{n+1}(X)=0 \text { for all } n \geqq 2 \text {. }
$$

Remark 1.10. If we consider the case $m=0$, we will obtain better results (cf. Theorem 2.4 and Corollary 2.5) than those given by Theorem 1.6 and Corollary 1.9.

\section{The "best" universal bound}

The previous section provides an upper bound for the order of some $k$-invariants of iterated loop spaces; our objective now is to discuss the prime factorisation of this bound. We start with the simply connected case.

THEOREM 2.1. Let $m \geqq 1$ and $n \geqq 2$ be given integers. For each prime number $p \leqq \frac{n-m}{2}+1$, there exists an m-connected infinite loop space $X$ such that its $k$-invariant $k^{n+1}(X)$ is a cohomology class of order $p$ in $H^{n+1}\left(X[n-1] ; \pi_{n} X\right)$.

Proof. Let $p$ be a prime $\leqq(n-m) / 2+1$, and let us define $s:=n-2 p+3$; notice that $n>s \geqq m+1 \geqq 2$. The Steenrod operation $P^{1}: H^{s}(-; \mathbb{Z} / p) \rightarrow$ $H^{n+1}(-; \mathbb{Z} / p)$ is a stable cohomology operation, i.e. corresponds to an infinite loop map $K(\mathbb{Z} / p, s) \rightarrow K(\mathbb{Z} / p, n+1)$; if $p=2$ we interpret $P^{1}$ as $S q^{2}$. The fibre $X$ of this map is an $(s-1)$-connected (with $s-1 \geqq m$ ) infinite loop space with only two non-trivial homotopy groups. Its unique $k$-invariant is the cohomology class corresponding to $P^{1}$ in $H^{n+1}(K(\mathbb{Z} / p, s) ; \mathbb{Z} / p)$; it is non-trivial since $s \geqq 2$.

Remark 2.2. We know from Theorem 1.6 that the integer $S_{n-m}$ is an upper bound for the order of the $k$-invariant $k^{n+1}(X)$ of all $m$-connected $r$-fold loop spaces $X$, assuming $2 \leqq n \leqq r+2 m$. For simply connected spaces, Theorem 2.1 provides the desired answer to the question we discuss in this section: if $m \geqq 1$, then the upper bound $S_{n-m}$ is the "best possible" in the following sense: if a prime $p$ divides $S_{n-m}$, there exists an $m$-connected $r$-fold loop space $X$ (actually an $m$-connected infinite loop space $X$ ) with $k^{n+1}(X)$ of order $p$.

We call this bound the "best possible", because we shall see later (cf. Remark 3.2 ) that, if we consider a $k$-invariant of finite order, we are essentially interested in the prime factors of its order (and not in the order itself).

Now it remains to look at the case $m=0$, for which the statement of Theorem 1.6 is the following: if $X$ is a connected $r$-fold loop space (with $r \geqq 2$ ), then $S_{n} k^{n+1}(X)=0$ for $2 \leqq n \leqq r$. But here the universal bound $S_{n}$ is not the "best possible". For example, if $X$ is a connected double loop space, Theorem 1.6 (with $\left.n=2, S_{2}=2\right)$ asserts that its first $k$-invariant $k^{3}(X) \in H^{3}\left(K\left(\pi_{1} X, 1\right) ; \pi_{2} X\right)$ sat- 
isfies $2 k^{3}(X)=0$; but we show in [3] that in fact

$$
k^{3}(X)=0 \text {. }
$$

The main result of [3] is a generalisation of this: let $n$ be an integer such that $n / 2+1$ is a prime number, and assume that $X$ is a connected $n$-fold loop space such that $\pi_{i} X=0$ for $1<i<n$ (i.e. $X[n-1]=K\left(\pi_{1} X, 1\right)$ ), then the first non-trivial $k$-invariant of $X$ is $k^{n+1}(X) \in H^{n+1}\left(K\left(\pi_{1} X, 1\right) ; \pi_{n} X\right)$ and it follows from Lemma 1.2 and the proof of Theorem 1.6 that $M_{n} k^{n+1}(X)=0$, but the conclusion of [3] is

$$
\left(M_{n} /\left(\frac{n}{2}+1\right)\right) k^{n+1}(X)=0 .
$$

This enables us to modify the assertion of Theorem 1.6 when $m=0$. Recall that a prime $p$ divides $S_{n}$ if and only if $p \leqq n / 2+1$.

Definition 2.3. For $n \geqq 2$,

$$
\bar{S}_{n}:=\left\{\begin{array}{l}
S_{n} /\left(\frac{n}{2}+1\right), \quad \text { if } \frac{n}{2}+1 \text { is a prime, } \\
S_{n}, \quad \text { otherwise. }
\end{array}\right.
$$

A prime number $p$ divides $\bar{S}_{n}$ if and only if $p<n / 2+1$ (for instance $\bar{S}_{2}=1$ ); therefore the product of all prime divisors of $\bar{S}_{n}$ is $M_{n-1}$.

THEOREM 2.4. If $X$ is a connected $r$-fold loop space (with $r \geqq 2$ ), then

$$
\bar{S}_{n} k^{n+1}(X)=0
$$

for $2 \leqq n \leqq r$.

Proof. It is sufficient to consider integers $n$ for which $p:=n / 2+1$ is a prime; thus we have $M_{n} / p=M_{n-1}$ and

$$
\bar{S}_{n}=S_{n} / p=M_{n} S_{n-1} / p=M_{n-1} S_{n-1} .
$$

Since $n \leqq r, X$ is a connected $n$-fold loop space, i.e. $X \simeq \Omega^{n} Y$ for some $n$-connected $C W$-complex $Y$. Let us define $G:=\pi_{1} X \cong \pi_{n+1} Y$ and $M:=$ $\pi_{n} X \cong \pi_{2 n} Y$. The $k$-invariants $k^{n+1}(X) \in H^{n+1}(X[n-1] ; M)$ and $k^{2 n+1}(Y) \epsilon$ $H^{2 n+1}(Y[2 n-1] ; M)$ are related by

$$
\left(\sigma^{*}\right)^{n}\left(k^{2 n+1}(Y)\right)=k^{n+1}(X),
$$

where $\left(\sigma^{*}\right)^{n}$ is the $n$-fold iterated cohomology suspension $[9$, p. 438].

Now let $\phi$ denote the Postnikov section $Y[2 n-1] \rightarrow Y[n+1]=K(G, n+1)$ and $F$ the fibre of $\phi$. The space $F$ has only finitely many non-trivial homotopy groups: $F$ is $(n+1)$-connected and $\pi_{i} F=0$ for $i>2 n-1$. It then follows from Lemma 1.4 and the universal coefficient theorem that $S_{n-1} H^{2 n+1}(F ; M)=0$. The Serre spectral sequence of this fibration gives us the exact sequence

$$
H^{2 n+1}(K(G, n+1) ; M) \stackrel{\phi^{*}}{\longrightarrow} H^{2 n+1}(Y[2 n-1] ; M) \longrightarrow H^{2 n+1}(F ; M) .
$$

Consequently, $S_{n-1} k^{2 n+1}(Y)$ belongs to the image of $\phi^{*}$; let $x$ be an element of 
$H^{2 n+1}(K(G, n+1) ; M)$ satisfying $\phi^{*}(x)=S_{n-1} k^{2 n+1}(Y)$. Let us look at the commutative diagram

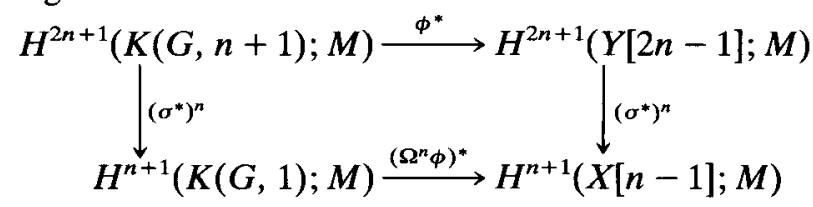

We have proved in [3, Proposition 3.1] that $M_{n-1}\left(\sigma^{*}\right)^{n}(x)=0$ in $H^{n+1}(K(G, 1)$; $M)$. Therefore we conclude:

$$
\begin{aligned}
\bar{S}_{n} k^{n+1}(X) & =M_{n-1} S_{n-1} k^{n+1}(X)=M_{n-1} S_{n-1}\left(\sigma^{*}\right)^{n}\left(k^{2 n+1}(Y)\right) \\
& =M_{n-1}\left(\sigma^{*}\right)^{n}\left(\phi^{*}(x)\right)=\left(\Omega^{n} \phi\right)^{*}\left(M_{n-1}\left(\sigma^{*}\right)^{n}(x)\right)=0 .
\end{aligned}
$$

COROLlaRY 2.5. If $X$ is a connected infinite loop space, then

$$
\bar{S}_{n} k^{n+1}(X)=0 \text { for all } n \geqq 2 .
$$

The next result asserts that the integer $\bar{S}_{n}$ involved in Theorem 2.4 is the "best possible" upper bound (in the same sense as above) for the order of the $k$-invariant $k^{n+1}(X)$ when $X$ ranges over all connected $r$-fold loop spaces, assuming $2 \leqq n \leqq r$.

THEOREM 2.6. Let $n \geqq 2$ be a given integer. For each prime number $p$ dividing $\bar{S}_{n}$, there exists a connected infinite loop space $X$ such that its $k$-invariant $k^{n+1}(X)$ is a cohomology class of order $p$ in $H^{n+1}\left(X[n-1] ; \pi_{n} X\right)$.

Proof. Let $p$ be a prime dividing $\bar{S}_{n}$ and let us define $s:=n-2 p+3$. Since $p<n / 2+1$, we have the inequality $s \geqq 2$ and we may use the method introduced in the proof of Theorem 2.1 in order to construct a connected infinite loop space $X$ with a unique $k$-invariant $k^{n+1}(X)$ of order $p$ in $H^{n+1}\left(X[n-1] ; \pi_{n} X\right)$.

\section{Relationships between algebraic $\boldsymbol{K}$-theory and linear group homology}

The $k$-invariants of a connected simple $C W$-complex $X$ explain, in some sense, how the space $X$ may be built up, up to homotopy type, out of EilenbergMacLane spaces: the vanishing of $k^{n+1}(X)$ in $H^{n+1}\left(X[n-1] ; \pi_{n} X\right)$ implies, for instance, the existence of a homotopy equivalence $X[n] \simeq X[n-1] \times K\left(\pi_{n} X, n\right)$ and, therefore, of a map $f: X \rightarrow K\left(\pi_{n} X, n\right)$ which induces an isomorphism on $\pi_{n} X$; a direct consequence of this is that the Hurewicz homomorphism $H u: \pi_{n} X \rightarrow H_{n}(X ; \mathbb{Z})$ is split injective (cf. also [6, Corollary 1.3]).

The purpose of this section is to generalise these results: the fact that a space $X$ has a $k$-invariant of finite order may also help us to understand the Hurewicz homomorphism $H u: \pi_{*} X \rightarrow H_{*}(X ; \mathbb{Z})$. In particular, we obtain information on this homomorphism when $X$ is an iterated loop space.

Lemma 3.1. Let $X$ be a connected simple $C W$-complex and assume that the $k$-invariant $k^{n+1}(X)$ is a cohomology class of finite order $s$ in $H^{n+1}(X[n-1]$; $\left.\pi_{n} X\right)$. Then

(a) there exists a map $f: X \rightarrow K\left(\pi_{n} X, n\right)$ such that the induced homomorphism $f_{*}: \pi_{n} X \rightarrow \pi_{n} X$ is multiplication by 
(b) the Hurewicz homomorphism $H u: \pi_{n}(X ; \mathbb{Z}[1 / s]) \rightarrow H_{n}(X ; \mathbb{Z}[1 / s])$ is split injective.

Proof. Part (a) is proved in [2, Lemma 4]. Because the map $f$ induces an isomorphism $f_{*}: \pi_{n}(X ; \mathbb{Z}[1 / s]) \stackrel{\longrightarrow}{\longrightarrow} \pi_{n}\left(K\left(\pi_{n} X, n\right) ; \mathbb{Z}[1 / s]\right)$, the composition

$$
\pi_{n}\left(X ; \mathbb{Z}\left[\frac{1}{s}\right]\right) \stackrel{H u}{\longrightarrow} H_{n}\left(X ; \mathbb{Z}\left[\frac{1}{s}\right]\right) \stackrel{f_{*}}{\longrightarrow} H_{n}\left(K\left(\pi_{n} X, n\right) ; \mathbb{Z}\left[\frac{1}{s}\right]\right)
$$

is also an isomorphism and $H u$ maps $\pi_{n}(X ; \mathbb{Z}[1 / s])$ injectively onto a direct summand of $H_{n}(X ; \mathbb{Z}[1 / s])$ : this gives (b).

Remark 3.2. When one considers a $k$-invariant of finite order, it is now clear, in view of assertion (b) of this lemma, that it is actually sufficient to know all prime divisors of its order (the exact order itself is not necessary for the applications developed in this section).

If $X$ is an $m$-connected space (with abelian fundamental group if $m=0$ ), the Hurewicz homomorphism $H u: \pi_{m+1} X \rightarrow H_{m+1}(X ; \mathbb{Z})$ is an isomorphism. If we restrict our attention to iterated loop spaces, it is then easy to deduce the following result on the Hurewicz homomorphism in higher dimensions.

THEOREM 3.3. If $X$ is an $m$-connected $r$-fold loop space (with $m \geqq 0, r \geqq 0$, $m+r \geqq 2$ ), then

(a) the Hurewicz homomorphism $H u: \pi_{n}\left(X ; \mathbb{Z}\left[1 / M_{n-m}\right]\right) \rightarrow H_{n}\left(X ; \mathbb{Z}\left[1 / M_{n-m}\right]\right)$ is split injective for $m+2 \leqq n \leqq r+2 m$, if $m \geqq 1$;

(b) $H u: \pi_{n}\left(X ; \mathbb{Z}\left[1 / M_{n-1}\right]\right) \rightarrow H_{n}\left(X ; \mathbb{Z}\left[1 / M_{n-1}\right]\right)$ is split injective for $2 \leqq n \leqq r$, if $m=0$.

Proof. Observe that $\mathbb{Z}\left[1 / S_{n-m}\right]=\mathbb{Z}\left[1 / M_{n-m}\right]$ and $\mathbb{Z}\left[1 / \bar{S}_{n}\right]=\mathbb{Z}\left[1 / M_{n-1}\right]$, since $M_{n-m}$ (respectively $M_{n-1}$ ) is the product of all primes dividing $S_{n-m}$ (respectively $\bar{S}_{n}$ ). According to the previous lemma, assertions (a) and (b) follow directly from Theorems 1.6 and 2.4 .

Finally, let $R$ be a ring with identity, $G L(R)$ its infinite general linear group, $E(R)$ the subgroup of $G L(R)$ generated by elementary matrices and $S t(R)$ the infinite Steinberg group of $R$. By performing the plus construction on the classifying spaces of these groups, we obtain the spaces $B G L(R)^{+}, B E(R)^{+}$and $B S t(R)^{+}$. Recall that $B E(R)^{+}$is the universal cover of $B G L(R)^{+}$(cf. [5, Proposition 1.1.7]) and that the canonical homomorphism $S t(R) \rightarrow E(R)$ whose kernel is $K_{2} R$ induces a fibration $B S t(R)^{+} \rightarrow B E(R)^{+}$whose fibre is $K\left(K_{2} R, 1\right)$ (cf. [5, Théorème 1.3.5]); in particular $B S t(R)^{+}$is 2-connected and $\pi_{n} B S t(R)^{+} \cong$ $\pi_{n} B E(R)^{+}$for $n \geqq 3$.

The algebraic $K$-theory of $R$ is defined by $K_{n} R:=\pi_{n} B G L(R)^{+}$, for $n \geqq 1$; notice that $K_{n} R \cong \pi_{n} B E(R)^{+}$if $n \geqq 2$ and $K_{n} R \cong \pi_{n} B S t(R)^{+}$if $n \geqq 3$. One has clearly: $K_{1} R \cong H_{1}(G L(R) ; \mathbb{Z}), \quad K_{2} R \cong H_{2}(E(R) ; \mathbb{Z}) \quad$ and $\quad K_{3} R \cong H_{3}(S t(R) ; \mathbb{Z})$. Because the spaces $B G L(R)^{+}, B E(R)^{+}$and $B S t(R)^{+}$are infinite loop spaces [8], the previous theorem describes the relations between algebraic $K$-theory and homology, in higher dimensions:

CoRollary 3.4. (a) The Hurewicz homomorphism Hu: $K_{n}\left(R ; \mathbb{Z}\left[1 / M_{n-1}\right]\right) \rightarrow$ 
$H_{n}\left(G L(R) ; \mathbb{Z}\left[1 / M_{n-1}\right]\right)$ is split injective for $n \geqq 2$;

(b) $H u: K_{n}\left(R ; \mathbb{Z}\left[1 / M_{n-1}\right]\right) \rightarrow H_{n}\left(E(R) ; \mathbb{Z}\left[1 / M_{n-1}\right]\right)$ is split injective for $n \geqq 3$;

(c) $H u: K_{n}\left(R ; \mathbb{Z}\left[1 / M_{n-2}\right]\right) \rightarrow H_{n}\left(S t(R) ; \mathbb{Z}\left[1 / M_{n-2}\right]\right)$ is split injective for $n \geqq 4$.

\section{References}

1 M. Arkowitz and C. R. Curjel. The Hurewicz homomorphism and finite homotopy invariants. Trans. Amer. Math. Soc. 110 (1964), 538-551.

2 D. Arlettaz. On the homology of the special linear group over a number field. Comment. Math. Helv. 61 (1986), 556-564.

3 D. Arlettaz. The first $k$-invariant of a double loop space is trivial (preprint).

4 H. Cartan. Algèbres d'Eilenberg-MacLane et homotopie (Séminaire H. Cartan Ecole Norm. Sup. 1954/1955).

5 J.-L. Loday. $K$-théorie algébrique et représentations de groupes. Ann. Sci. Ecole Norm. Sup. (4) 9 (1976), 309-377.

6 J.-P. Meyer. Whitehead products and Postnikov systems. Amer. J. Math. 82 (1960), 271-280.

7 R. Thom. L'homologie des espaces fonctionnels. Colloque de Topologie Algébrique, Louvain (1956), 29-39.

8 J. B. Wagoner. Delooping classifying spaces in algebraic $K$-theory. Topology 11 (1972), 349-370.

9 G. W. Whitehead. Elements of Homotopy Theory. Graduate Texts in Math 61 (New York: Springer, 1978).

(Issued 16 December 1988) 